\title{
What if you could only publish 50 papers your entire career?
}

\author{
David C. Muddiman ${ }^{1}$
}

Published online: 16 December 2015

(C) Springer-Verlag Berlin Heidelberg 2015

Ethical questions in science are often centered on the veracity, accuracy, and expediency of results in published articles. A majority of the support for academic studies is sponsored by taxpayers, resulting in an obligation to disseminate the research, a notion supported by the proliferation of Library of Congress journals, eJournals, and publically available reports. Yet a majority of publications have little impact, resulting in few or no peer citations, or are not even read by other scientists in the same field, resulting in the duplication of funding for questions that have already been effectively addressed. This begs the question, if a mechanism were in place to reduce the pressure to publish, would these problems be marginalized?

What if you could only publish 50 papers your entire career as corresponding author? This translates mathematically to $2 \%$ of your scientific footprint per manuscript. Would you change the depth of your inquiry, rigor of experimental design and data interpretation, and the clarity in which you put your work into the context of the literature? Ultimately, would your conclusions propel your field forward in a significant way?

Regardless of journal type or impact factor, each manuscript should detail a complete and significant study. The effort required to publish with a clear conceptual framework-from experimental design to evidence-based positive or negative conclusions - goes beyond that of many papers submitted to meet a minimal publishable unit

David C. Muddiman

dcmuddim@ncsu.edu

1 Department of Chemistry, North Carolina State University, Campus Box 8204, Raleigh, NC 27695-8204, USA
(MPU). Though this shift to the highest publishable unit (HPU) will not mean every paper is a blockbuster, it would guarantee that each manuscript fulfills one or more of three goals:

1. Demonstrates extraordinary innovation and creativity

2. Critically details facets of new workflows and technologies

3. Provides key insights and mechanisms into a meaningful application

With the proliferation of online and niche journals, there is near limitless access to publish any work. Yet both our personal and public reputations as scientists can be rapidly diluted if, for example, only one in 100 papers has significant depth and influence in the field.

The philosophical question posed in this editorial runs counter to the "publish or perish" line of thinking that drives us to constantly, and almost instinctively, submit the MPU. However, it is my sincere belief that shying away from a number system and almost corporate-like model of success will enrich our scientific community. Equally important, as corresponding authors, we have an opportunity to change expectations and model new behavior for the next generation of scientists, who often emerge cynical about academic publishing, and excite them about the prospect of pursing science in its purist form. In spite of the financial incentives offered by industry, renewing an emphasis on the pure pursuit of scientific research, supported by changing publication guidelines and attitudes, will draw talent and creativity to the field.

Though a 50-paper limit may merely be a theoretical exercise to help re-evaluate our publication process, it is useful in defining the characteristics and process for submitting a manuscript that meets the goals of having the "clear conceptual framework." 


\section{Guidelines to justify the submission of a "50-paper" limit manuscript}

Reflection: A successful experiment does not make a research article. An article should only be prepared when the following questions may be answered "YES." Am I proud of this study? Does the study clearly convey a new perspective or contribution? Does this study add significant value to the intended field?

Justification: Conviction must be expressed in the form of a cover letter and also included in the introduction and/or conclusion. Can you present a clear and concise case that this study is important?

Presentation: Evidence-based conclusions must be clearly outlined in the manuscript. Do your methods justify the results? Did you demonstrate the new approach by measuring the analytes in a highly idealized sample or real samples? Can your results be corroborated using state-of-the-art (bio)analytical methods and statistics? Did you discover or disprove something really important?

Acknowledgment: Validation of the work through peer review. Did you suggest effective reviewers? Are they experts who will be highly critical (but fair)? Do they encompass a breadth of applications that allow them to make a value judgement on your research? Can they find critically important elements that can improve your paper?

In summary, whether the limit is 25 or 250 papers, I hope that critical thought on this topic will lead to an increase in the impact of publications. The impact that is, for example, quantitated by the $h$-index and the $i 10$ index, which attempts to balance the landscape. If we all thought more deeply about the research we are submitting, and moved that bar from the MPU to the HPU, all other things that result would be pleasant, including innovation, external funding, awards and most important, impact!

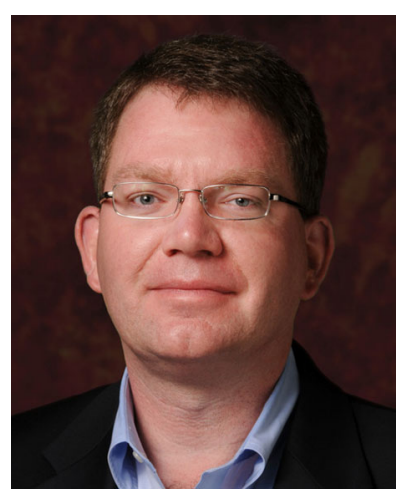

David C. Muddiman is the Jacob and Betty Belin Distinguished Professor of Chemistry and Founder and Director of the W.M. Keck FTMS Laboratory for Human Health Research at North Carolina State University in Raleigh, NC. Prior to moving his research group to North Carolina State University, he was a Professor of Biochemistry and Molecular Biology, and Founder and Director of the Mayo Proteomics Research Center at the Mayo Clinic College of Medicine in Rochester, MN. Dr. Muddiman is Editor of Analytical and Biological Chemistry and Associate Editor of the Encyclopedia of Analytical Chemistry as well as on the Editorial Advisory Board of Mass Spectrometry Reviews, Molecular and Cellular Proteomics, Rapid Communications in Mass Spectrometry, and the Journal of Chromatography B. He also serves on the Advisory Board of the NIH Funded Complex Carbohydrate Research Center, University of Georgia, and the Yale/NIDA Neuroproteomics Center, Yale University. He is currently a member of the ASMS Board of Directors and is the Treasurer and member of the Executive Board of US-HUPO and recently was elected as President of US HUPO. His group has published over 225 peer-reviewed papers and has received four US patents. He is the recipient of the 2015 ACS Award in Chemical Instrumentation, 2010 Biemann Medal, American Society for Mass Spectrometry, 2009 NCSU Alumni Outstanding Research Award, the 2004 ACS Arthur F. Findeis Award, the 1999 American Society for Mass Spectrometry Research Award, and the 1990-91 Safford Award, University of Pittsburgh, for Excellence in Teaching. 